www.jmscr.igmpublication.org
Impact Factor 3.79

ISSN (e)-2347-176x

crossref DOI: http://dx.doi.org/10.18535/jmscr/v3i9.42

\title{
Inferior Vena Caval Thrombosis in Elderly Patients with Active Pulmonary Tuberculosis: A Case Series with Implications for Therapy.
}

Authors

\section{Afshan Shabir ${ }^{1}$, Parvaiz Koul ${ }^{2}$}

Department of Internal Medicine, Sheri Kashmir Institute of Medical Sciences, Srinagar, Kashmir, India Email:drafshanshabir@gmail.com

\begin{abstract}
Introduction: Prevalence of tuberculosis continues to remain high worldwide According to 2010 Global Burden of Disease estimates, majority of tuberculosis related deaths occurred in people older than 50. Tuberculosis (TB) is associated with a hypercoagulable state and hence VTE. These can be extensive and potentially life threatening. A variety of factors have been postulated to predispose patients with tuberculosis to venous thrombosis that include local stasis due to venous compression by lymph nodes and relative immobility caused by respiratory dysfunction, alteration in coagulation factors and reactive thrombocytosis. Case series presentation: We report 2 cases of extensive venous thromboembolism associated with pulmonary tuberculosis in the elderly. A 65 year-old Asian female with recently diagnosed tuberculosis presented with a new long deep vein thrombus extending from tibial vein up to right heart chamber and 67 year old Asian male in whom VTE was a presenting feature of tuberculosis (CFV thrombus extending up to $I V C)$. Inferior vena caval thrombosis has been reported only rarely in active pulmonary tuberculosis and only 3 reports exist in English literature

Conclusion: Our two cases highlight the risk of deep venous thrombosis in elderly patients with pulmonary tuberculosis even in the absence of any other risk factors for VTE. TB should be considered as a risk factor for $V T E$ and included in thromboembolism risk evaluation especially in first few months of treatment. VTE might be the first presentation of underlying tuberculosis. Also, VTE associated with tuberculosis might be extensive and involve proximal veins hence, may be life threatening due to a higher risk of pulmonary embolism. Physicians must be aware of this for prompt diagnosis and treatment to prevent fatality. Patients on tubercular therapy might require high doses of anticoagulation due to known interaction
\end{abstract}

\section{Introduction}

Tuberculosis persists as a major cause of morbidity and mortality worldwide. As per WHO Global tuberculosis report 2014, in 2013 an estimated 9 million people developed tuberculosis and 1.5 million died from the disease $($ (WHO), 2014). According to 2010 Global Burden of Disease estimates, the majority of tuberculosis-related deaths occurred among people older than 50; most in those aged 65 and above. Tuberculosis can rarely present with venous thrombosis which is rooted in the development of a transient hypercoagulable state in a subset of patients (Saturnino Suárez Ortega, 1993). White et al in a 
retrospective South African study reported that the prevalence of DVT in tuberculosis is $3.4 \%$ within first two weeks after initiation of antitubercular therapy due to endothelial dysfunction by rifampicin ${ }^{(\mathrm{NW}, 1989)}$. However a more recent Italian study reported the prevalence to be $0.3 \%$ in the first month of starting antibercular therapy, nearly all occurring in hospitalized patients (Ambrosetti M, 2006). Link between haemostatic changes and TB related pro-thrombotic state normalizes with an adequate ATT ${ }^{\text {(Turken } \mathrm{O}, 2002)}$. A variety of factors have been postulated to predispose patients with tuberculosis to venous thrombosis that include local stasis due to venous compression by lymph nodes and relative immobility caused by respiratory dysfunction (Ambrosetti M, 2006) (Turken O, 2002) (Robson SC, 1996), alteration in coagulation factors and reactive thrombocytosis (Robson SC, 1996). The haemostatic changes leading to a prothrombotic state are reported to normalize following treatment of the underlying infection (Turken O, 2002).

Despite the fact that India is host to a huge burden of tuberculosis, there are only few reports of venous thrombosis in tuberculosis from India. These have included venous thrombosis in lower limb veins,Inferior vena cava,portal vein,cerebral veinsand retinal vein (Parvaiz A Shah, 2011) (Prasad Muley, 2014) (Rahul Naithani, 2007) (Kakkar N, 2003) (Verma R, 2013) (Jyoti Kumar, 2013) (Mithun Raj, 2006).

We herewith report 2 cases of inferior vena caval thrombosis (IVC) in patients with evidence of active pulmonary tuberculosis.

\section{Case 1}

65 year-old Asian female presented to Emergency department (ED) with painful left leg swelling of 7 day duration. Patient gave no history of smoking or alcohol intake. She was non-diabetic and normotensive. Previously diagnosed Pulmonary Tuberculosis 2 months ago based on high ADA (137), lymphocytic exudative pleural effusion, positive Montoux test, acid-fast bacilli (AFB) in sputum (>30bacilli/field) and high ESR (60). She was commenced on DOTS Category 2 (Isoniazid 300mg, Rifampicin 450mg, Pyrazinamide 1500mg, Ethambutol 750mg).General physical examination revealed thin female body mass index of 26. She was apyrexial, haemodynamically stable, $\mathrm{SPO}_{2}(\mathrm{FiO} 2$ 21\%) was 97\%. Systemic examination of chest, cardiovascular and abdominal system was unremarkable. Left leg was swollen and tender on palpation. Arterial blood gas analysis (Fio2 21\%) was normal with pO2 $98 \mathrm{mmHg}$, pCO2 28, ph 7.45, $\mathrm{HCO} 319.5, \mathrm{Na}^{+}$ $139, \mathrm{~K}^{+}$4.1.Routine investigations revealed normocytic anaemia (Hb $9.3 \mathrm{~g} / \mathrm{dl}$ ), MCV 85, leucocyte $8180 / \mu \mathrm{l}$ and platelet were normal (Bilirubin 0.3, ALT 20, and ALP104). Ultrasound left leg demonstrated deep vein thrombus of anterior and posterior tibial vein extending into popliteal vein, common femoral vein, ileal vein, inferior vena cava up to right heart chamber.Echocardiogram showed no right atrium or ventricular thrombus, normal systolic function (Ejection fraction 70\%). CECT chest, abdomen and pelvis showed mild pleural effusion with a normal abdomen/pelvis. No other risk factors for VTE identified. Baseline INR was 1.6 and APTT40.5. Thrombophilia profile was not sent due to financial constraints. Patient was commenced on therapeutic heparin and warfarin. She required $13 \mathrm{mg}$ of warfarin to achieve a therapeutic INR and was discharged home after 10 days with plan of follow up in pulmonary clinic.

IMAGE Ultrasound Doppler shows thrombus involving lefttibial vein, popliteal vein, femoral vein, ileal vein and inferior vena cava

\section{Case 2}

A 67 year old Asian male, smoker, non-diabetic and normotensive presented to ED with painful right lower limb swelling of 3 day duration. He had 3 months history of fever, shortness of breath and productive cough. There was no history of haemoptysis or alcohol intake. His brother was treated for pulmonary TB 6 months ago. General physical examination revealed a malnourished man with body mass index of 24 . He was febrile (Temp $100^{\circ} \mathrm{F}$ ), Pulse rate $106 \mathrm{bpm}, \mathrm{BP}$ 126/80 $\mathrm{mmHg}$ and $\mathrm{Sp02} 90 \%$ (FiO2 21\%). Chest 
auscultation revealed bilateral rhonchi. Cardiovascular, abdominal and neurological examination was normal. The right leg was swollen and tender to touch. Venous blood gas $\mathrm{Na}^{+} 146, \mathrm{~K}^{+} 3.5, \mathrm{Ph} 7.38, \mathrm{PCO} 238, \mathrm{HCO} 322.5$. Laboratory findings on admission revealed Haemoglobin $11 \mathrm{~g} / \mathrm{d}$, normal WBC count, liver function, renal function and bone profile tests. Baseline INR was 1. Chest X-ray demonstrated bilateral infiltrates and cavitation. Bilateral lower limb Ultrasound Doppler was done and confirmedright common femoral vein (CFV) and saphenous vein (SFV) thrombus extending to infra-renal Inferior vena cava (IVC) Contrast CT chest/abdomen confirmed multifocal lung consolidation with cavitations and thrombus extending from ileal vein into right infra-renal IVC. Echocardiogram demonstrated normal left ventricular function, no pericardial effusion. CT pulmonary Angiogram (CTPA) ruled out pulmonary embolism. No other risk factors for VTE identified. Sputum was positive for AFB (>30 bacilli/field). Patient commenced on standard ATT and therapeutic heparin with warfarin. He achieved therapeutic INR on $6 \mathrm{mg}$ of warfarin. His general state improved on treatment and was discharged after 2 weeks with plan of follow-up at pulmonary clinic.

IMAGE CECT abdomenthrombus extending from ileal veininto right infra-renal IVC

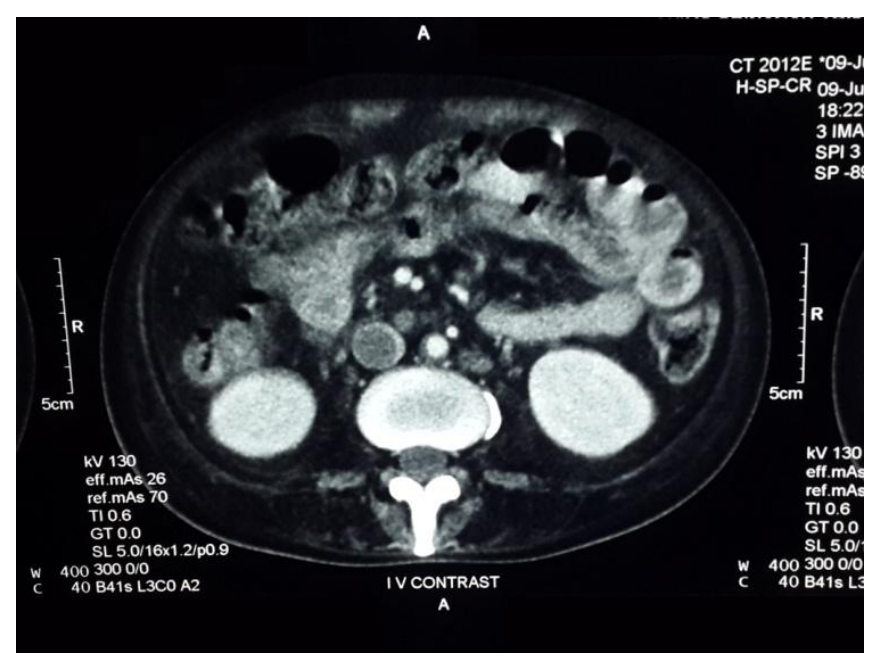

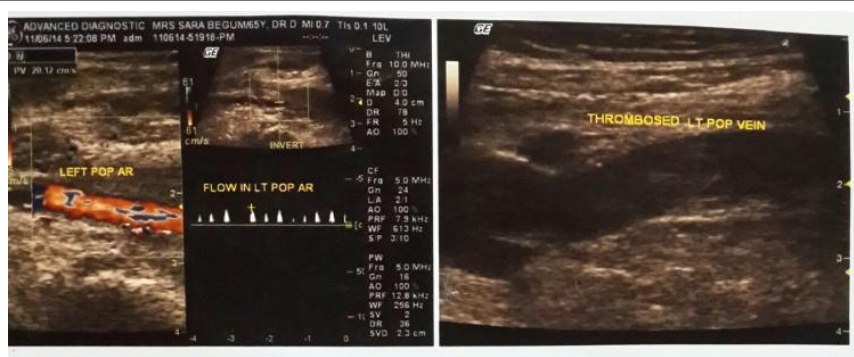

Image 1

Image 2
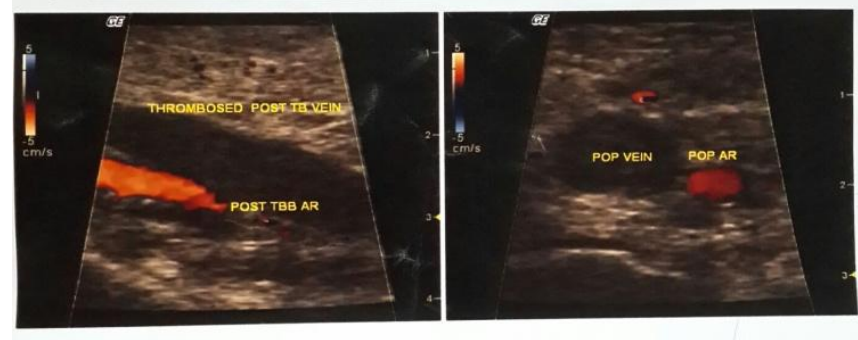

Image 3

Image 4
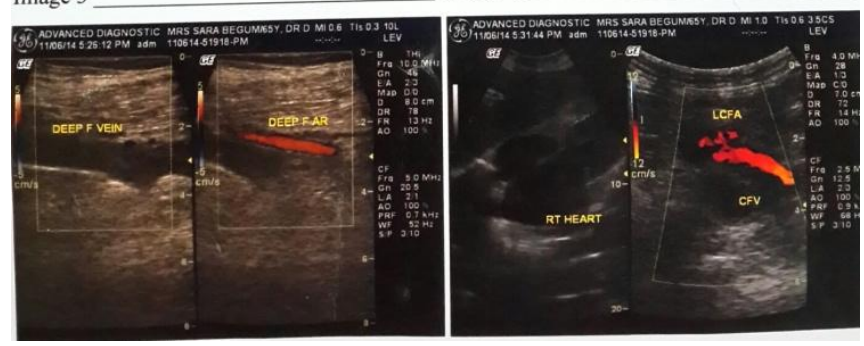

\section{Discussion}

Both of our patients had extensive inferior vena caval thrombosis which was the presenting manifestation in one patient and occurred within 2 months of initiation of antitubercular therapy in the other. Inferior vena caval thrombosis has been reported only rarely in active pulmonary tuberculosis and only 3 reports exist in English literature.

The hypercoagulablity in tuberculosis has been postulated to arise from a number of factors like reduced antithrombin III and protein C levels, elevated plasma fibrinogen levels, and an increased aggregation of platelets (Turken O, 2002) (Robson SC, 1996). Serum fibrinogen rises within the first 2 weeks of therapy and subsequently normalizes within 12 weeks. Coupled with impaired fibrinolysis,elevated fibrinogen levels may result in a prothrombotic state ${ }^{\text {(Robson SC, 1996). }}$ Additionally endothelial dysfunction may result in the systemic inflammatory response to tuberculosis and combined with subtle changes in blood rheologic properties (Kaminskaia GO, 1997) may result in intravascular coagulation. A higher 
frequency of antiphospholipid antibodies (Saturnino Suárez Ortega, 1993) and Protein $\mathrm{S}$ deficiency has been reported in tuberculosis. Protein $\mathrm{S}$ deficiency may result from an increase in concentration of $\mathrm{C} 4 \mathrm{~b}$ binding protein an acute phase reactant which binds protein $\mathrm{S}$ in plasma, and a production of IL1 and TNF-aby peripheral blood mononuclear cells that can result in down regulation of protein $\mathrm{C} /$ protein $\mathrm{S}$ during any sepsis (Manuel Casanova-Román, ${ }^{2002)}$. Studies have also demonstrated a possible association between deep venous thrombosis and use of rifampicin with a relative risk of 4.74 in patients treated with rifampicin containing regimens. This does not contraindicate the use of this drug in patients at risk, but such patients need close monitoring.Also,retroperitoneal adenopathy may cause inferior vena cava thrombosis in absence of any haemostatic abnormality (A Gogna, 1999). Higher dose of warfarin is necessary to achieve therapeutic INR levels due to rifampicin effect on cytochrome P450 (T H Self, 1975).

\section{Conclusions}

Our two cases highlight the risk of deep venous thrombosis in elderly patients with pulmonary tuberculosis even in the absence of any other risk factors for VTE. TB should be considered as a risk factor for VTE and included in thromboembolism risk evaluation especially in first few months of treatment. VTE might be the first presentation of underlying tuberculosis. Also, VTE associated with tuberculosis might be extensive and involve proximal veins hence, may be life threatening due to a higher risk of pulmonary embolism. Physicians must be aware of this for prompt diagnosis and treatment to prevent fatality. Patients on tubercular therapy might require high doses of anticoagulation due to known interaction.

\section{Consent}

Written informed consent was obtained from the patients for publication of these case series and any accompanying images. A copy of the written consent is available forreview by the Editor-inChief of this Journal.

\section{Competing interests}

The authors declare that they have no competing interests.

\section{References}

1. World Health Organization (WHO), "Global tuberculosis report," 2014.

2. Artiles Vizcaíno,Balda Aguirre,Melado Sánchez, Arkuch Saade,Ayala Galán Saturnino Suárez Ortega, "Tuberculosis as risk factor for venous thrombosis," An Med Interna, vol. 10, no. 8, pp. 398-400, 1993.

3. White NW, "Venous Thrombosis and rifampicin," Lancet, vol. 2, pp. 434-435, 1989.

4. Ferrarese M, Codecasa L, Besozzi G, Sarassi A, Viggiani P, Migliori G Ambrosetti M, "Incidence of Venous Thromboembolism in Tuberclosis Patients," Respiration, vol. 73, p. 396, 2006.

5. Kunter E, Solmazgul E, Cerrahoglu K, Ilvan A Turken O, "Hemostatic Changes in active Pulmonary Tuberclosis," international journal of tuberculosis and lung disease, vol. 6, no. 10, pp. 927-932, 2002.

6. White NW, Aronson I, Woolgar R, Goodman H, Jacobs P Robson SC, "Acutephase Response And The Hypercoagulable State in Pulmonary Tuberclosis," British Journal of Hematology, vol. 93, pp. 943949, 1996.

7. Yawar Yaseen, Abdul H Malik Parvaiz A Shah, "Pulmonary Tuberculosis with Deep Venous Thrombosis," General Medicine, Case Report 2011.

8. Urjita Shah, Varsha Shah, Dulari Gandhi Prasad Muley, "Deep Vein Thrombosis With Tuberculosis: A Rate Presentation of Common Disease," GLOBAL JOURNAL OF MEDICINE AND PUBLIC HEALTH, vol. 3, no. 1, 2014. [Online]. www.gjmedph.org 
9. Neerja Agrawal, Ved P Choudhary Rahul Naithani, "Deep venous thrombosis associated with tuberculosis," Blood Coagulation \& Fibrinolysis, vol. 18, no. 4, pp. 377-380, June 2007.

10. Banerjee AK, Vasishta RK, Marwaha N, Deodhar SD Kakkar N, "Aseptic Cerebral Venous Thrombosis Associated With Abdominal Tuberculosis," Neurol India, vol. 51, no. 1, pp. 128-129, 2003.

11. Lalla R, Patil TB, Tiwari N Verma R, "A Rare Presentation of Cerebral Venous Sinus Thrombosis Associated With Tubercular Meningitis.," BMJ Case Rep PMC3761660, 2013.

12. Veena Chowdhury, K Rajeshwari Jyoti Kumar, Arab Journal of Gastroenterology, vol. 14, no. 2, pp. 85-86, June 2013.

13. Agarwal A Mithun Raj, "Inferior Vena Cava Thrombosis Complicating uberculosis," The Newzeland Medical Journal, vol. 119, no. 1244, p. U2279, Oct 2006.

14. Serebrianaia BA, Martynova EV, Mishin VI. Kaminskaia GO, "Intravascular Coagulation As a Typical Concomitant of Acute Pulmonary Tuberculosis," Problemy Tuberkuleza, vol. 3, pp. 42-46, 1997.

15. Jesús Rios, Antonio Sánchez-Porto, and Manuel Casanova-Bellido Manuel Casanova-Román, "Deep Venous Thrombosis Associated with Pulmonary Tuberculosis and Transient Proteins Deficiency," Scandinavian Journal of Infectious Diseases, vol. 34, no. 5, pp. 393-394, 2002.

16. G R Pradhan, R S K Sinha, B Gupta A Gogna, "Tuberculosis Presenting As Deep Vein Thrombosis," Postgraduate Medical Journal, vol. 75, pp. 104-106, 1999.

17. $\mathrm{R}$ B Mann $\mathrm{T} H$ Self, "Interaction of Rifampin And Warfarin," Chest, vol. 67, no. 4, pp. 490-491, 1975. 